\title{
Main Exploration on Technical Measures for Machinery and Equipment Lightweight Component
}

\author{
Yi Jiang ${ }^{1, a}$, Miao $\mathrm{He}^{2}$, Li Xu \\ ${ }^{1,2,3}$ Nanchang Institute of Science and Technology, 330108 \\ ajiangyijx@126.com
}

Keywords: Machinery equipment; Lightweight component; Technical measures

\begin{abstract}
Machinery equipment lightweight component weighs a lot in machinery equipment design and production. It can efficiently improve equipment's performance in environment-protection and energy-saving. Researches and progress in lightweight component has drawn extensive concern from many experts in China and abroad along with the growing tendency of domestic machinery industry. Till now, the main way to obtain the lightweight component is to build materials performance metrics database, level up the design standard and upgrade technical skills. This paper includes these three aspects above.
\end{abstract}

\section{Introduction}

The lightweight mechanical equipment component is one of the topics facing the machinery and equipment design and manufacture and also one of the important means to improve the performance of products. The lightweight mechanical equipment components can realize not only energy saving and emission reduction, resource conservation, improving resource utilization and increasing the benefits, but also the establishment of an environmental protecting, sustainable developing society with the improving and modification of machinery equipment design ${ }^{[1]}$. The basic principle of lightweight components is to ensure the advanced function, safety and reliability of mechanical equipment by improving the design, material, and other technologies. Accordingly, appropriate technical means are meant to realize the lightweight mechanical equipment component. This paper mainly discusses the main ways to achieve this goal.

\section{Current Situation of Chinese Machinery Equipment Lightweight Component}

The requirements for lightweight component in China's market are still very strict. Comparing to ordinary components, lightweight component is required to ensure safety and reliability in use on one hand. On the other hand, it also means to reduce production cost, improve working efficiency and realize energy saving and environmental protection, which is a big challenge in mechanical equipment component design and manufacturing technology. In addition, large machinery manufacturing industry in China mainly adopts measures of a selection of high quality materials, optimizing the product design and the using of advanced skills for mechanical equipment lightweight components, especially in equipment manufacturing, automobile industry, aerospace, military and other major engineering field. In recent years, the construction and development of the engineering field for lightweight machinery the equipment component has brought more development opportunities and good prospects.

\section{Material Performance Index for Lightweight Component}

Usually the mechanical equipment will show different strength requirements under different stress states including stress state of symmetric bending cyclic stress, simple bending, simple tensile and simple torsion ${ }^{[2]}$. These four kinds of stress state is an important condition for the measurement of mechanical equipment materials components and material properties to determine mechanical equipment lightweight components according to the performance of lightweight materials in the test. Comparing the index of lightweight performance with the lightweight alloy indexes such as iron, 
aluminum, titanium and magnesium, a lightweight component material index and relevant database can be established and correspondent equipment component can be figured out. That is to say, an important prerequisite and basis for mechanical equipment design of lightweight component is to establish mechanical equipment components of lightweight materials. Performance test results show that under different force state, there are also differences the performance index of material. But the regularity conclusion is that the strength index of component material quality and component per unit length is in negative related relationship under the same condition of bearing capacity.

\section{Design of Lightweight Component}

What underlies mechanical equipment lightweight component design is a combination of the influence from outside elements including temperature and time on the material property and specific analysis on the stress state of components, the resistance index and failure mode. Materials under different stress states, temperatures, even high temperature corrosion will present different property index of lightweight materials. According to the different indexes and research results, we can establish the mechanical equipment lightweight component material performance index system and the corresponding database. With the database comparing, different material strength index researching, and specific needs of mechanical equipment including, there comes the practical design of mechanical equipment lightweight component ${ }^{[3]}$.

\section{Discussion on Modification Process of Lightweight Component}

The improvement of producing technology of mechanical equipment component is important to realize the lightweight of mechanical components. Following aspects are the mainly discussed.

(1) An overall performance development of material can be realized in an advance in mechanical equipment component technical skill under a premise of stable component material density, especially an improvement in the strength of materials. In this way can reduce the overall weight of the structure without a change in the total load on the components of machinery and equipment.

(2) By using the improved technology of the components of the mechanical equipment, the section shape of the component can be improved, and the bending resistance (bending section coefficient) and torsion resistance (section modulus in torsion) of the member section can be improved $^{[4]}$. The improvement of this index can achieve a reduction of mechanical components total weight by strengthening the bearing capacity without changing the total load of the mechanical components.

(3) Improving the process of mechanical equipment and component technology can improve the mechanical equipment component surface strength, thereby strengthening the combat element under different loading conditions. In general, the lightweight of the mechanical components is realized by improving the surface resisting index of the equipment components.

(4) The use of various technology like rolling, shot blasting and the others can strengthen the mechanical equipment component surface strength, help the organization to strengthen the material surface, and thus increase the total level of application of load component surface, while reducing the overall weight of the component.

In a word, the main way to realize lightweight components is firstly analyzing, studying and comparing different material properties in different stress states, then establishing material property index accordingly. Instructed by this index, lightweight component can be realized by employing advanced skills to increase the materials' flexing resistance, buckling resistance, surface bearing capacity and strength.

\section{Conclusion}

Performing as an important issue in machinery equipment production, lightweight component stands at a key position in machinery design and production around every business ${ }^{[5]}$. The premise 
of light weight of mechanical components is to ensure the advanced performance and performance stability of the equipment component. Equipment in the service period should also ensure safety and reliability, which is the basic principle of lightweight components design equipment. This paper mainly expounds the basic principles of the lightweight mechanical components and discusses the main technical way to realize it. To put it in a specified way, first, the performance index system and database of the system need to be established according to the performance index of the material under different conditions and stress state. Then conduct lightweight component design accordingly and improve lightweight technical skills to get to the final point.

In recent years, China's modern mechanical manufacturing industry has developed rapidly and the level of scientific and technological progress has improved, the mechanical lightweight components has become a prominent problem to be solved in manufacturing field. In order to promote the realization of this goal, it is necessary to strengthen the exchange of technology and theory, introduce advanced technology, and constantly promote the reform and scientific innovation to promote its efficient development.

\section{Reference}

[1] Qunpeng Zhong, Yiliang You, Zheng Zhang. Discussion on Main Technical Methods of Machinery Equipment Lightweight Component[J]. Journal of Mechanical Engineering, 2012, 48(18):2-6;

[2] Zhi Yu. Discussion on Main Technical Methods of Machinery Equipment Lightweight Component[J]. Heilongjiang Science and Technology Information, 2013, 12(17):95-95;

[3] Zhiyun Pu. Discussion on Main Technical Methods of Machinery Equipment Lightweight Component[J]. Science \& Wealth, 2013, 15(7):332-332;

[4] Chinese Mechanical Engineering Society. Journal of Mechanical Engineering. Strengthening Science and Technology Innovation, and Actively Promote the Rapid Development of Lightweight Equipment Components[J]. Journal of Mechanical Engineering, 2012, 48(18):1.

[5] Zhiwen Liu, Luoxing Li. Review of Bending Short Process for Lightweight Component[J]. Transactions of Nonferrous Metals Society of China, 2014, 24(8):2003-2012. 